\title{
Enhanced Microsphere Transport in Capillary by Conditioned Cells of Green Paramecia Used as Living Micromachines Controlled by Electric Stimuli
}

\author{
Shunsuke Furukawa and Tomonori Kawano* \\ Laboratory of Chemical Biology and Bioengineering, \\ Faculty and Graduate School of Environmental Engineering, \\ The University of Kitakyushu, Kitakyushu 808-0135, Japan
}

(Received June 10, 2011; accepted October 21, 2011)

Key words: BioMEMS, green paramecia, micro-biorobotics, particle transport

Some researchers have described the cells of Paramecium species as "swimming sensory cells" or "swimming neurons" applicable to micro-biorobotics and biological micro-electromechanical systems (BioMEMS). Paramecium species including green paramecia (Paramecium bursaria) migrate towards the anodic electrode when exposed to an electric field. This type of cellular movement is known as galvanotaxis. Because the ideal micromachines designed for microparticle transport must have a capacity for loading certain numbers of particles, $P$. bursaria was chosen as a model organism. In this study, we show enhanced microparticle transport by overcoming (i) the particle size limitation for the cell-mediated transport of microspheres of up to $c a$. $10 \mu \mathrm{m}$ size (doubling the size of particles ever reported) and (ii) the limit of cellular migration distance manifested by galvanotactically stimulated cells.

\section{Introduction}

To biologists, Paramecium species are very familiar laboratory tools for cell biological and environmental studies. ${ }^{(1)}$ Cells of Paramecium species are now considered as model systems for studying cellular signal transduction mechanisms. ${ }^{(2)}$ Since signal perception, processing and reactions are completed within these unicellular organisms, some researchers have described the cells of Paramecium species as "swimming sensory

*Corresponding author: e-mail: kawanotom@kitakyu-u.ac.jp 
cells"(3) or "swimming neurons".(4)

Over a century ago, it was shown that Paramecium species exhibit galvanotaxis, since paramecia align with an electric field or voltage gradient and swim toward the anode if the electric field is sufficiently strong.(5) It is well known that Paramecium species including green paramecia (Paramecium bursaria) migrate towards the anodic electrode when exposed to an electric field in a medium. This type of cellular movement is known as galvanotaxis. Our previous study revealed that an electric stimulus applied to green paramecia is converted to a galvanotactic cellular movement with the involvement of the T-type calcium channel on the plasma membrane. ${ }^{(6)}$ The above studies indicate the possibility for finely geared neuronal controls and engineering of unicellular micromachineries. In fact, the galvanotactic responsiveness observed in Paramecium species (particularly P. caudatum) has attracted the attention of bioengineers in the fields of biorobotics, microrobotics or biological micro-electromechanical systems (BioMEMS) in order to develop electrically controllable micromachineries. ${ }^{(7-9)}$

While the ideal micromachines designed for microparticle transport must be equipped with a certain capacity for loading particles, Paramecium caudatum, one of the wellstudied galvanotactically controllable species, ${ }^{(3,4,7-9)}$ lacks such characteristics. Therefore, from this viewpoint, $P$. caudatum should be replaced with a related species with greater particle-loading capacity.

A single green paramecium cell, the swimming vehicle of interest, naturally harbors several hundred cells of the endosymbiotic green algae within its cytosol, which is morphologically and genetically almost identical to Chlorella species. ${ }^{(10)}$ Recent studies have demonstrated that the abundant symbiotic algae inside the host cells can be replaced, after the forced removal of the algae, ${ }^{(11)}$ with various artificial particles such as fluorescent and magnetic microspheres. ${ }^{(12,13)}$ Instead of inert particles, several living organisms such as photosynthetic bacteria (a strain derived from Synechocystis sp. PCC $6803)^{(14,15)}$ or nonsymbiotic algae ${ }^{(16)}$ can also be introduced. Thus, this organism provides a highly advanced model for evolutionary emergence of photosynthetic organisms through the development of symbiosis..$^{(17)}$

It is tempting to apply the above characteristics of green paramecia, namely, particle internalization and swimming capability, to micro-biorobotic demonstration. In the micro-biorobotic approach, Aonuma et al. ${ }^{(6)}$ carried out the quantification of galvanotactic migration of $P$. bursaria in the open top bath. Using P. bursaria, Furukawa et al. ${ }^{(12)}$ demonstrated for the first time that the electrically driven transports of two distinct types of small particles differed in size, namely, nanosized particles and microsized particles (microspheres) in the capillary system. The sizes (diameters) of polystyrene microspheres labeled with fluorescent dyes or magnetite, previously transported using galvanotactically controlled green paramecia, were $2.28,2.88$, and $5.6 \mu \mathrm{m}$, ${ }^{(12)}$ by analogy to the size of the cells of endosymbiotic algae (between 2 and $5 \mu \mathrm{m}$ ) which are native to $P$. bursaria's intracellular space.

In this study, we show enhanced microparticle transport by challenging and overcoming (i) the particle size limitation for the cell-mediated transport of microspheres of up to $c a$. $10 \mu \mathrm{m}$ size (doubling the size of particles ever reported) and (ii) the limit of migration distance manifested by galvanotactically stimulated cells. In addition, an attempt to control cellular swimming using magnetic particles is also described. 


\section{Materials and Methods}

\subsection{Paramecium cell line}

The green paramecium (P. bursaria) strain INA-1 (Fig. 1; syngen 1, mating type I) was originally collected from the studying point INA ${ }^{(18)}$ on Ongagawa River (Kama City, Fukuoka Prefecture, Japan). Since the cell line was established after single cell isolation, all the cells in the culture were clones sharing identical genetic background. The cells were propagated and maintained in a modified culture medum as previously reported. ${ }^{(19)}$ Briefly, the culture medium was prepared with a yeast-extract-based nutrition tablet (1 EBIOS tablet/l; Asahi Food \& Healthcare, Tokyo, Japan). The culture medium was renewed at 2-week intervals. One nutrition tablet $(250 \mathrm{mg})$ contains $94.2 \%(\mathrm{w} / \mathrm{w})$ dried yeast homogenates and $5.5 \%(\mathrm{w} / \mathrm{w})$ carbohydrates. The bacterized nutrition medium was prepared by inoculating the medium with the food bacterium Klebsiella pneumoniae $1 \mathrm{~d}$ prior to the subculture of ciliate cells. The ciliate culture was initiated with $c a$. 10-20

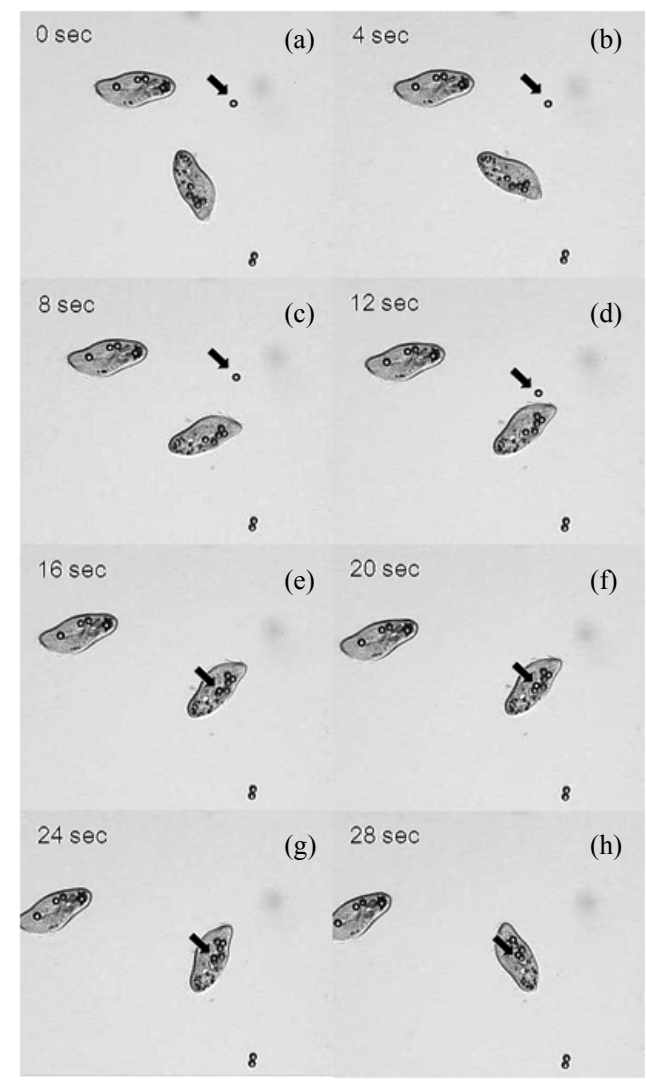

Fig. 1. Uptake of a microsphere by a freely swimming conditioned cell of green paramecia $(P$. bursaria). Microspheres of $5.6 \mu \mathrm{m}$ diameter were added to the culture of conditioned P. bursaria. Every $4 \mathrm{~s}$, an image was taken. Arrow, target microsphere to be taken up by the swimming cell. 
cells $/ \mathrm{ml}$ and propagated to the confluent level (over $1000 \mathrm{cells} / \mathrm{ml}$ ) under a light cycle of $12 \mathrm{~h}$ light and $12 \mathrm{~h}$ dark with $c a .3000$ lux ( $30 \mathrm{~cm}$ from the light source) of fluorescent natural-white light at $23^{\circ} \mathrm{C}$.

\subsection{Conditioned cells}

Conditioned cells of $P$. bursaria (INA-1 cells), which are ready for particle loading, were prepared by the forced removal of the algal symbiont from the hosting ciliate as described by Tanaka et al. ${ }^{(11)}$ Briefly, the green cells were incubated in the presence of $0.1 \mu \mathrm{M}$ paraquat for over $24 \mathrm{~h}$ under light (with a fluorescent natural-white light, 3000 lux at least). Then, a single ciliate lacking algae was separated under a microscope and the cell line of apo-symbiotic paramecia derived from this single cell was propagated for comparison in the lettuce infusion inoculated with food bacteria as described above. The resultant white cells are ready for particle feeding as demonstrated in Fig. 1.

\subsection{Microspheres}

Polystyrene microspheres (diameters of 3.87, 5.60, 7.32, 9.75, and $15.45 \mu \mathrm{m}$ ) and magnetic microspheres (diameter, $2.88 \mu \mathrm{m}$; COMPELTM Uniform Magnetic Microspheres) were all obtained from Bangs Laboratories, Inc. (Fishers, IN, USA). Information on the particle size was provided by the vendor and it was confirmed by flow particle image analysis using FPIA-2100 (data not shown), according to the procedures described elsewhere. ${ }^{(12,13)}$

\subsection{Cyanobacterial cells}

Among cyanobacteria, the material to be loaded to the conditioned P. bursaria cells, Synechocystis sp. PCC 6803 was chosen since it is one of the most extensively studied strains. ${ }^{(15)}$ PCC 6803 cells were propagated in BG-11 medium under continuous light at $c a .3000$ lux with fluorescent natural-white light at $23^{\circ} \mathrm{C}$. Then, the conditioned $P$. bursaria cells were used for the introduction of PCC 6803 cells. Conditioned P. bursaria cells were incubated with a suspension of PCC 6803 cells $(c a .2000$ cells $/ \mathrm{ml})$ in lettuce infusion for $30 \mathrm{~min}$ in an Eppendorf tube at room temperature. The resultant PCC 6803-fed regreened cells were collected on a nylon mesh (pore size, $10 \mu \mathrm{m}$ ) and washed three times with $10 \mathrm{ml}$ of fresh medium. The PCC 6803-fed cells could be maintained and propagated for over 1 year. ${ }^{(14)}$

\subsection{Galvanotactic cell migration inside the capillary system}

As a platform of galvanotactic cell movements, polypropylene tubes (diameter, 1-2 mm) filled with culture medium were connected to $1.5 \mathrm{~V}$ battery cells with copper leads. No specific metal except for copper was used for preparing a pair of electrodes. For performing the migration of a cell population from one end of the tube to the other, galvanotaxis was allowed as follows. Prior to the application of electric charge, 100 cells of $P$. bursaria $(50 \mu \mathrm{l}$ of dense culture, $2000 \mathrm{cells} / \mathrm{ml})$ were injected to the cathodic end of the medium-filled capillary tube. The cellular movement was terminated by breaking the current with a switch. After exposure to $1.5 \mathrm{~V} \mathrm{DC}$, each capillary tube was cut into 4 fractions. Then, galvanotactically altered localizations of the cells were quantified 
by counting the cells under a stereomicroscope (SMZ645; Nikon, Tokyo, Japan) after transferring each fraction onto a depletion slide.

\subsection{Microscopic analysis}

Intact live cells were used for routine observations (cell counting) under a stereomicroscope (SMZ645; Nikon, Tokyo, Japan). For obtaining the digital microscopic images, the P. bursaria cells with and without symbiotic green algae were fixed in $3 \%$ $(\mathrm{w} / \mathrm{v})$ formaldehyde added to the culture medium and the fixation was allowed at room temperature for $5 \mathrm{~min}$. Microscopic images of the paramecium cells were acquired using a Radiance 2100 microscope (Bio-Rad Laboratories, Hercules, CA).

\subsection{Conductance measurement}

Resistance and conductance in the low-ion-strength culture medium were determined under $9 \mathrm{~V}$ using a handy conductivity meter (CD-5021A, Custom Co.Ltd., Tokyo, Japan).

\section{Results and Discussion}

\subsection{Phagocytosis-like incorporation of microspheres by conditioned cells}

The uptake and transport of microparticles can be readily observed by simply adding the microspheres $(5.60 \mu \mathrm{m}$ in diameter) to the suspension of conditioned P. bursaria cells (Fig. 1). Once the cell senses the presence of particles (resembling food bacteria in size), the cell migrated towards the particle and smoothly ingested the particle into the cells. Thus, loading of the particle was completed. While holding the particle inside the cell body, the cell started to move away; thus, the particle was transported.

These events can be routinely observed both in nature and in cultures in vitro. The key difference between natural and conditioned cells of P. bursaria is the capacity for particle uptake. As previously reported, ${ }^{(13)}$ a single cell of natural P. bursaria harbors several hundred cells of green algae; thus, by evacuating (conditioning) the cells, a great capacity for any particles of interest could be created. Into a single conditioned cell, multiple numbers of particles can be loaded depending on the size of the particles (Fig. 2). Among the microspheres we examined, the largest particles successfully taken up by the conditioned P. bursaria cells were the $9.75-\mu \mathrm{m}$-diameter microspheres. The cells failed to incorporate the $15.45-\mu \mathrm{m}$-diameter microspheres. We assume that the maximum size is between 9.75 and $15.45 \mu \mathrm{m}$.

\subsection{Galvanotactic transport of microparticles}

As shown in Fig. 3, assays of galvanotactic migration of microsphere-loaded cells were performed using capillary tubes. Here, the procedure for testing the galvanotactic cell migration from the cathodic side to the anodic side within the capillary is illustrated [Fig. 3(a)]. Prior to the application of electric charge, 100 cells were injected to the cathodic end of the capillary. After the cells were exposed to $1.5 \mathrm{~V}$, each capillary tube was cut into 4 fractions. Then, quantification of cell accumulation or migration was 
(a)

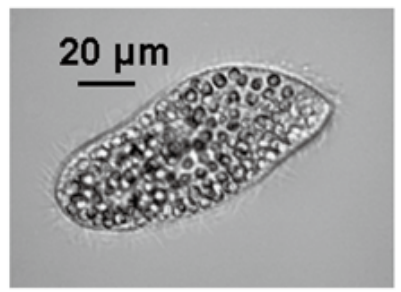

(c)

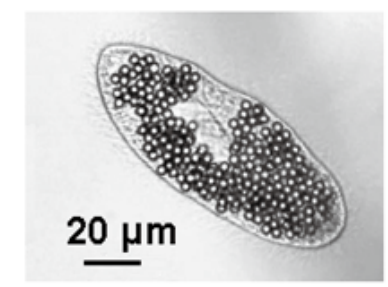

(e)

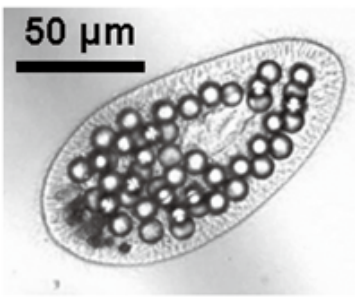

(b)

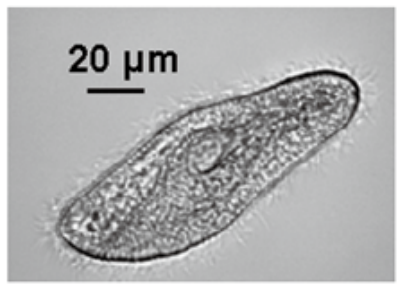

(d)

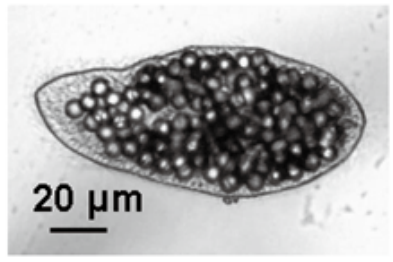

(f)

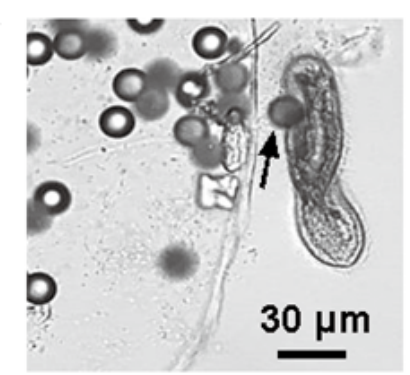

Fig. 2. Cells of P. bursaria with and without various types of particle. (a) A single cell of wildtype green paramecia harboring green algae as symbionts. (b) A conditioned cell ready for particle loading. Conditioned cells after loading of microspheres of (c) $3.87 \mu \mathrm{m}$ diameter, (d) $7.32 \mu \mathrm{m}$ diameter, and (e) $9.75 \mu \mathrm{m}$ diameter. (f) Microspheres of $15.45 \mu \mathrm{m}$ diameter left unloaded into the cells. Arrow indicates one of microsphere $(15.45 \mu \mathrm{m}$ diameter $)$ being stuck in the oral groove, suggesting the size limit to be taken up via the oral-mediated path.

carried out by counting the cells in each fraction. By this way, galvanotactic migration of conditioned cells in the 60 -mm-long capillary tube, after loading of $3.87-\mu \mathrm{m}$-diameter microspheres, 7.32- $\mu \mathrm{m}$-diameter microspheres and 9.75- $\mu \mathrm{m}$-diameter microspheres, were observed [Figs. 3(b)-3(d)].

Thus, now, we concluded that all the particles of up to $c a$. $10 \mu \mathrm{m}$ diameter can be transported by the swimming paramecia, which is almost double the size tested in earlier demonstrations. ${ }^{(12)}$

\subsection{Effect of magnet-magnet interaction on cellular migration}

Magnetically labeled microspheres $(2.88 \mu \mathrm{m}$ in diameter) were loaded into the conditioned $P$. bursaria cells and used for the galvanotactic migration assay. Here, we attempted the control of the flow of cells in the capillary by simply placing the 


\section{(b) $3.87 \mu \mathrm{m}$ beads}

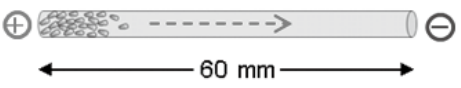

(a) Quantification of galvanotactic
cell migration in the capillary
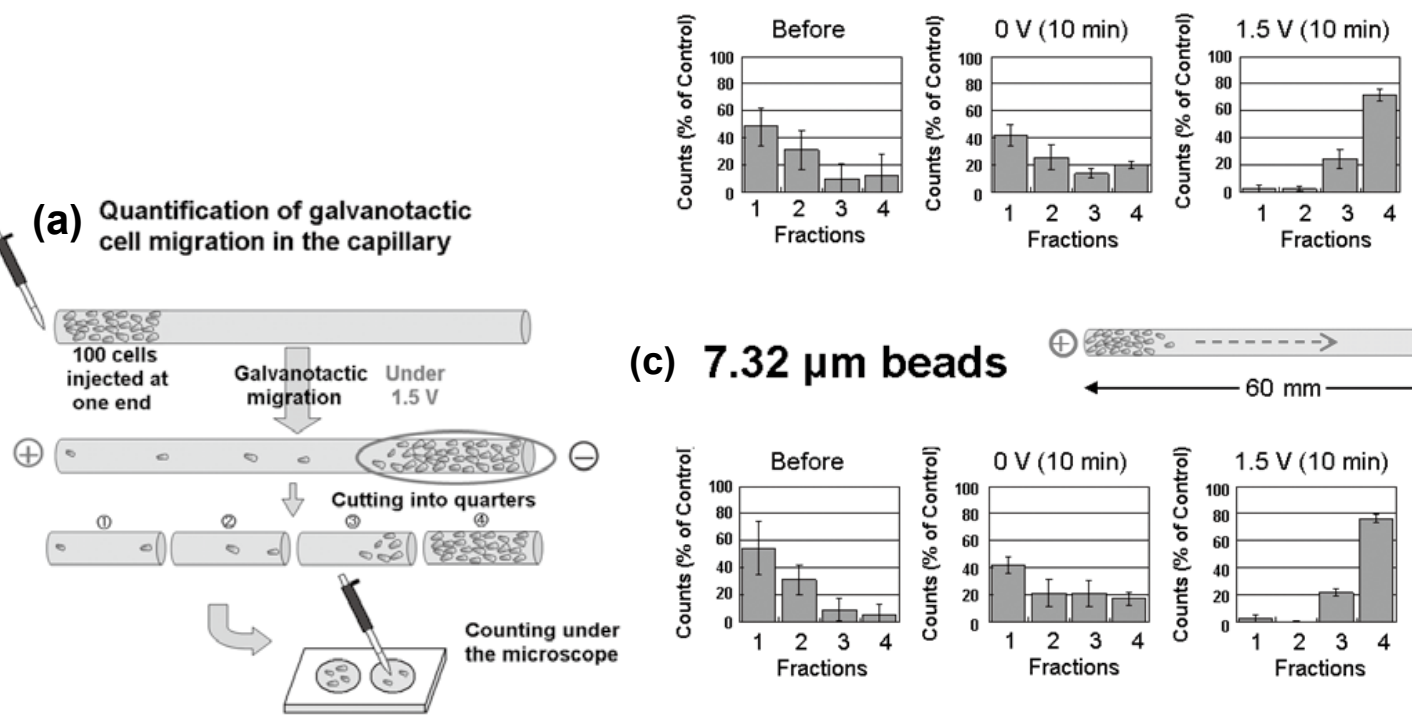

(c) $7.32 \mu \mathrm{m}$ beads
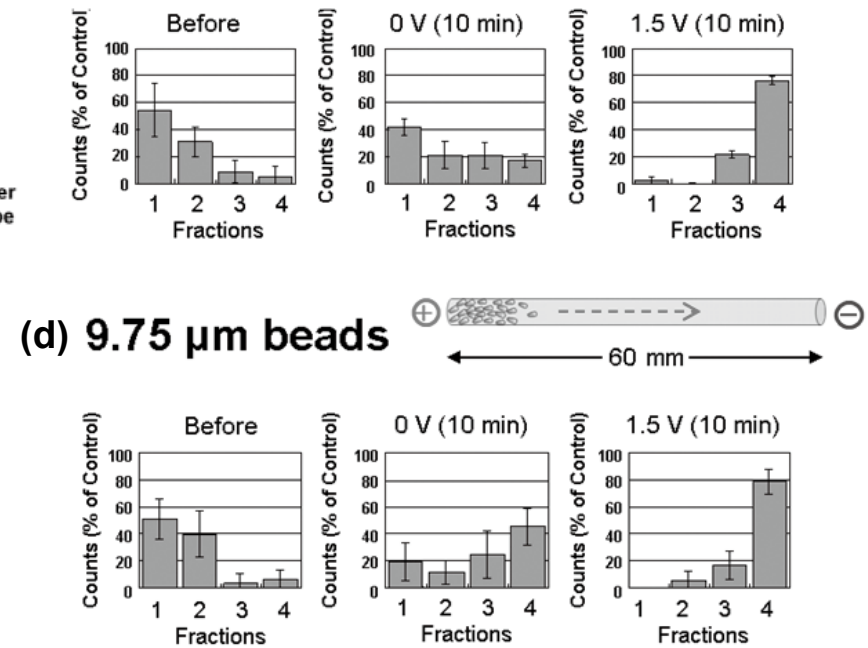

Fig. 3. Galvanotactic cell migration assays in capillary tubes. (a) Procedure for testing the galvanotactic cell migration from the cathodic side to the anodic side within the capillary. Prior to the application of electric charge, 100 cells were injected to the cathodic end of the capillary. After the cells were exposed to $1.5 \mathrm{~V}$, each capillary tube was cut into 4 fractions. Then, quantification of cell accumulation or migration was carried out by counting the cells in each fraction. Galvanotactic migration of conditioned cells in the 60-mm-long capillary tube, after loading of microspheres of (b) $3.87 \mu \mathrm{m}$ diameter, (c) $7.32 \mu \mathrm{m}$ diameter, (d) and $9.75 \mu \mathrm{m}$ diameter. Error bars in (b)-(d) represent S.D. $(n=4)$.

neodymium magnet (Fig. 4). As expected, cellular movement was significantly retarded by the cell trapping action of the magnet. This demonstration suggests that the electrically stimulated migration of magnetically labeled cells of $P$. bursaria can be negatively controlled under magnetic interference; thus, the timing of cell release under 

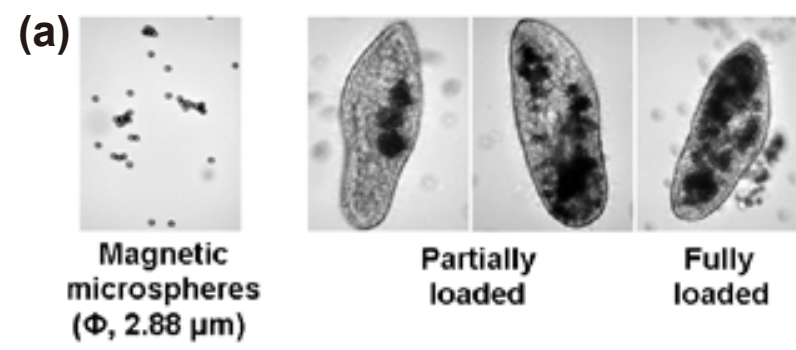

(b) Attracting magnetic bead-loaded cells by strong magnet

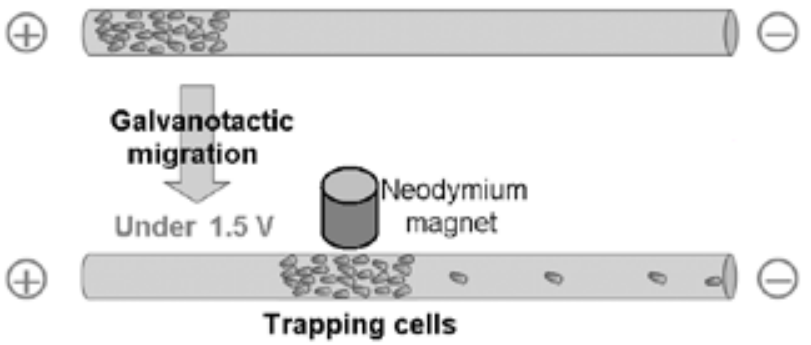

\section{(c) Retardation of galvanotactic cell migration by magnet-magnet interaction}
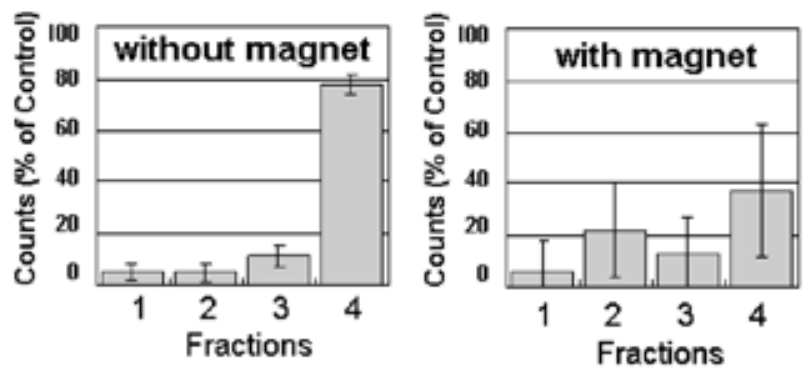

Fig. 4. Magnet-dependent retardation of the galvanotactic migration of $P$. bursaria cells harboring magnetic microspheres. To the conditioned cells of $P$. bursaria, magnetic microspheres of $2.88 \mu \mathrm{m}$ diameter were loaded and used for cell migration assays. The galvanotactic assays were carried out using 60-mm-long capillary tubes. (a) Magnetic microspheres and magnetic microsphereloaded cells of P. bursaria. (b) Design of the assay. A small neodymium magnet was placed at a region corresponding to the second fraction of the capillary. For comparison, assay without magnet was carried out as control. Except for the use of the magnet, the assay conditions were all identical to those described in Fig. 3. (c) Distribution of the cells in the capillary with and without the presence of the neodymium magnet. Error bars in (c) represent S.D. $(n=4)$. 
continuous electrical current or stopped-flow controls can be manifested on the basis of this technique. However, at present, some loss of cells due to the forced removal of magnetic particles out of the moving cells (thus, breaking the cellular membrane) under magnetic fields was observed. Therefore, more modifications are required prior to actual uses in BioMEMS.

\subsection{Galvanotactic transport of microbial cells}

In addition to the nonbiological particles, living microbial cells could be captured and transferred in the capillary system using the conditioned $P$. bursaria cells under electrical controls. A typical demonstration was carried out using cyanobacteria-loaded cells of P. bursaria (Fig. 5). Galvanotactic migration of microbe-loaded cells of P. bursaria, namely, electrically controlled transport of the cells of cyanobacteria (PCC 6803) packed in the swimming micromachines, was performed using the identical system described for the transport of microspheres. This demonstration implies that our micromachines can fetch and return with a bacterial harvest. The application of this system to microbial sampling and identification in the microenvironment is now being developed by our group.

\subsection{Long-distance migration under repeated electric stimuli}

The capillary tubes used for the above demonstrations were all $60 \mathrm{~mm}$ long, which is perhaps a very short distance for many readers; however, the above distance is $c a$. 600 times longer than the length of paramecium cells ( $c a .100 \mu \mathrm{m}$ in length). The distances between two electrodes used for galvanotactic assays using P. bursaria in previous reports ranged between 30 and $120 \mathrm{~mm} \cdot{ }^{(6,12)}$ In fact, over the recent 100 years, the migration distance performed by the electrically stimulated Paramecium species has been limited to within $c a$. $100-120 \mathrm{~mm}$ owing to technical difficulties. In fact, Paramecium species including $P$. bursaria favor low-ionic-strength media or water; therefore, there is a tendency that the electrical resistance between the two electrodes used for galvanotactic assays will become apparently high (over some ten mega-ohm). In the case of the medium used in this study, the resistance reached 16 and 33 mega-ohm at 120 and $240 \mathrm{~mm}$, respectively (Fig. 6). Thus, by doubling the migration distance, the resistance is also doubled. One of the likely approaches to realizing the long-distance migration of living P. bursaria cells is the application of higher voltages. However, this approach barely works since the cells of $P$. bursaria are highly sensitive and fragile under high voltage, and thus, readily burst out at around the electrodes, as previously reported. ${ }^{(6)}$ Under $1.5 \mathrm{~V}$, a galvanotactic cell movement assay using a 240 -mm-long tube was performed but the accumulation of the cells at the anodic end was barely observed even after $400 \mathrm{~min}$ of exposure to electricity [Fig. 7(a)].

Since the migration distance performed by living cells would be one of the key factors for designing the future application of cell-based BioMEMS or robotic systems, we tested a novel approach, the stepwise protocol [Fig. 7(b)]. Here, an attempt for longdistance galvanotactic migration of $P$. bursaria cells in $240-\mathrm{mm}$-long capillary tubes was performed by stepwisely setting the electrodes at moderate distances (in the present demonstration, $120 \mathrm{~mm}$ ). For the first pair of electrodes set at 0 and $120 \mathrm{~mm}$ from the 
(a)

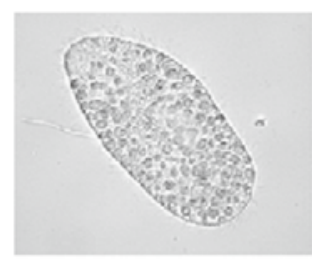

(c)

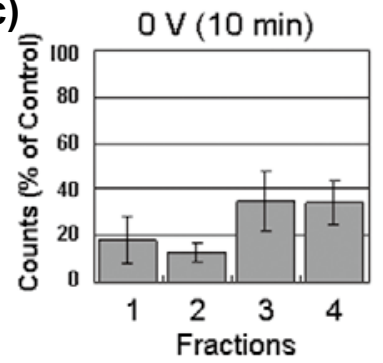

(b)

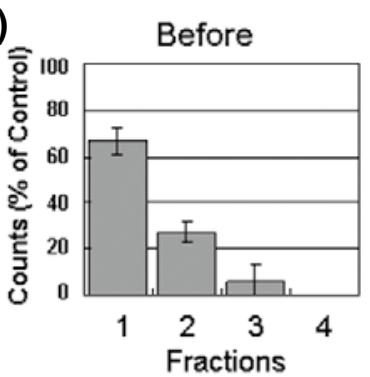

(d)

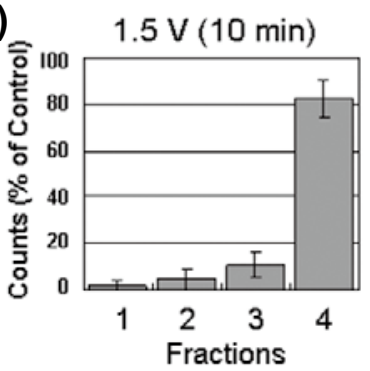

Fig. 5. Galvanotactic migration of cyanobacteria (PCC 6803)-loaded cells of P. bursaria. The conditions of the galvanotactic assays are identical to those described in Fig. 3. (a) Cyanobacteria (PCC 6803)-loaded cells of $P$. bursaria prepared according to the method of Ohkawa et al. ${ }^{(14)}$ (b) Distribution of cells in the capillary before application of electric stimulus. (c) $10 \mathrm{~min}$ after static incubation without electric stimulus. (d) After the cells were exposed to $1.5 \mathrm{~V}$ for $10 \mathrm{~min}$. Error bars in (b)-(d) represent S.D. $(n=4)$.

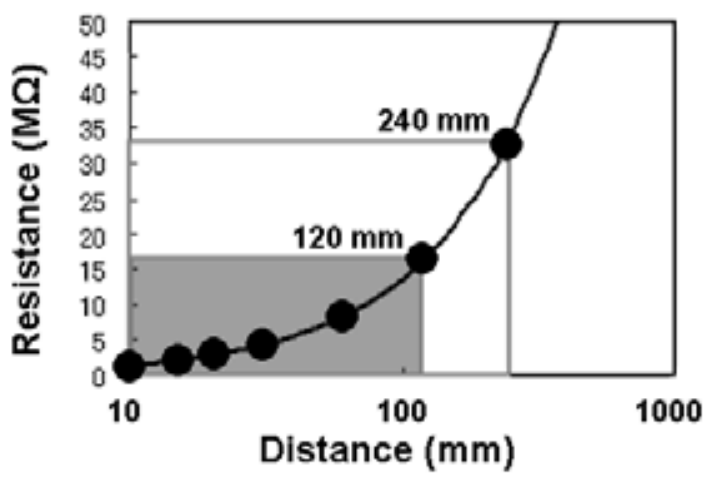

Fig. 6. Resistance increase with distance in the yeast-extract-based low-ionic strength culture/ assay medium for $P$. bursaria. 
(a)

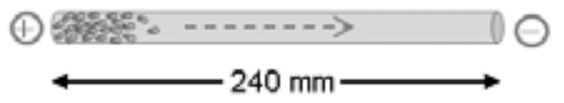

(b)

\section{Step 1.}

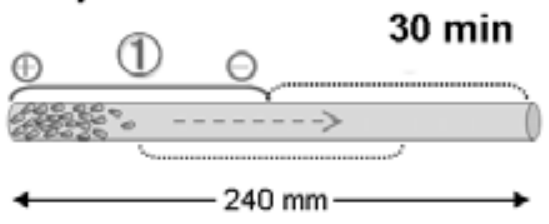

Step 2.

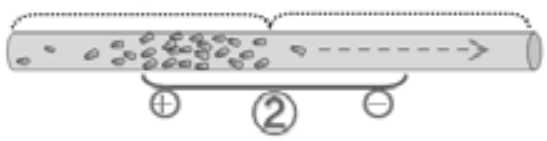

\section{Step 3.}

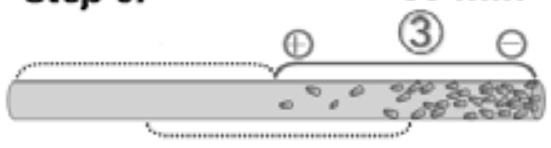

(c)

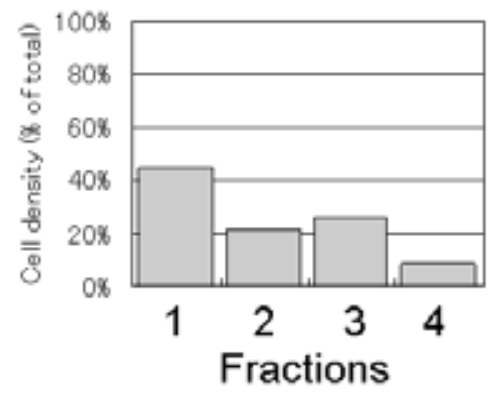

(d)

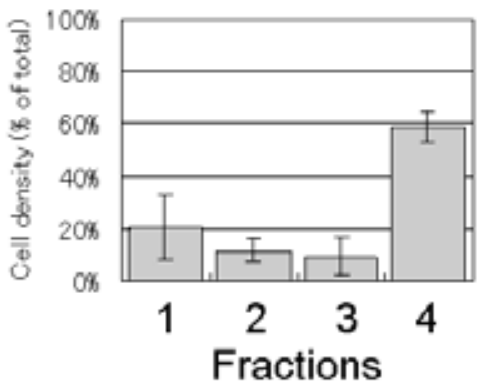

Fig. 7. An attempt for long-distance galvanotactic migration of P. bursaria cells. By stepwise application of a short-distance electric field, $P$. bursaria cells finally accumulated in the anodic fraction. (a, c) Effect of continuous galvanotactic stimulation (1.5 V, $400 \mathrm{~min}$ ), applied between electrodes $240 \mathrm{~mm}$ apart. (b, d) Stepwise cell migration driven by three-time-repeated 30-minlong electric stimuli $(1.5 \mathrm{~V})$ with $120 \mathrm{~mm}$ distance. Error bars in (c) and (d) represent S.D. $(n=4)$.

cathodic end, most cells were pushed forward to around the middle of the capillary tube. Then, a second pair of electrodes was applied to further push the cells toward the anodic side. Finally, a third pair of electrodes was used for finalizing the cell migration over a $240 \mathrm{~mm}$ distance. Each "electrophoresis" at $1.5 \mathrm{~V}$ lasted for $30 \mathrm{~min}$. Thus, within a total of $90 \mathrm{~min}$, the majority of the cells reached the anodic end. To achieve successful cell migration by the stepwise method, it is important to overlap the regions of repeated galvanotaxis as performed here.

To further enhance the long-distance migration of $P$. bursaria cells, $125-\mathrm{mm}$-long cell migration processes (under $1.5 \mathrm{~V}, 30 \mathrm{~min}$ ) were repeated 15 times to complete the 
1000-mm-long cell migration, spending a total of $7.5 \mathrm{~h}$ (data not shown). However, at each of the 15 migration steps, only 80 to $90 \%$ of the cells could be collected around the anode; thus, the number of lost cells accumulated at the end, and therefore, only $4.4 \%$ of the cells could be collected in the anodic quarter fraction. Probably, such loss at each of the repeated steps could be minimized by optimizing the time, ionic strength, and voltage (at nonlethal range), in future trials.

\subsection{Perspectives}

Here, we described the electrical controls of the cellular movement and particle transport using conditioned cells of P. bursaria. We are currently involved in the development of alternative approaches for micro-biorobotic controls such as the use of light signals emitted by a light-emitting diode.

\section{Acknowledgements}

This work was supported by a grant from the Kyushu Semiconductor Industries \& Technology Innovation Association (SIIQ) and a Grants-in-Aid for Scientific Research by Ministry of Education, Culture, Sports, Science and Technology (MEXT), Japan (Research Project Number: 23656495).

\section{References}

1 N. Miyoshi, T. Kawano, M. Tanaka, T. Kadono, T. Kosaka, M. Kunimoto, T. Takahashi and H. Hosoya: J. Health Sci. 46 (2003) 429.

2 L. L. Pech: Comp. Biochem. Physiol. A: Mol. Integr. Physiol. 111 (1995) 31.

3 H. Machemer and J. E. de Peyer: Deutsche Zool (Gesellschaft, Erlangen, 1977) pp. 86-110.

4 Y. Naitoh: Electrical Conduction and Behaviour in "Simple" Invertebrates, ed. G. A. B. Shelton (Clarendon Press, Oxford, 1982) pp. 1-48.

5 K. Ludloff: Arch. Gesamte Physiol. 59 (1895) 525.

6 M. Aonuma, T. Kadono and T. Kawano: Z. Naturforsch., C: Biosci. 62 (2007) 93.

7 A. Itoh: IEEE/ASME Trans. Mechatron. 5 (2000) 181.

8 N. Ogawa, H. Oku, K. Hashimoto and M. Ishikawa: Proc. IEEE Int. Conf. on Robotics and Automation (IEEE, New Orleans, 2004) pp. 1646-1651.

9 N. Ogawa, H. Oku, K. Hashimoto and M. Ishikawa: Proc. IEEE Int. Conf. on Robotics and Automation (IEEE, Barcelona, 2005) pp. 1258-1263.

10 T. Kadono, T. Kawano, H. Hosoya and T. Kosaka: Protoplasma 223 (2004) 133.

11 M. Tanaka, M. Murata-Hori, T. Kadono, T. Yamada, T. Kawano, T. Kosaka and H. Hosoya: Acta Protozool. 41 (2002) 255.

12 S. Furukawa, C. Karaki and T. Kawano: Z. Naturforsch., C: Biosci. 64 (2009) 421.

13 K. Irie, S. Furukawa, T. Kadono and T. Kawano: Z. Naturforsch., C: Biosci. 65 (2010) 681.

14 H. Ohkawa, N. Hashimoto, S. Furukawa, T. Kadono and T. Kawano: Plant Sig. Behav. 6 (2011) 773.

15 H. Ohkawa, H. B. Pakrasi and T. Ogawa: J. Biol. Chem. 182 (2000) 31630.

16 B. I. Gerashchenko, N. Nishihara, T. Ohara, H. Tosuji, T. Kosaka and H. Hosoya: Cytoometry $41(2000) 209$.

17 T. Kawano, T. Kadono, H. Hosoya and T. Kosaka: Z. Naturforsch., C: Biosci. 59 (2004) 538.

18 S. Nishihama, A. Haraguchi, T. Kawano, K. Michiki, K. Nakazawa, T. Suzuki, K. Uezu and K. Yoshizuka: Limnology 9 (2008) 35.

19 T. Kadono, K. Uezu, T. Kosaka and T. Kawano: Z. Naturforsch., C: Biosci. 61 (2006) 541. 